\title{
Gender Bias in Student Evaluations - Corrigendum
}

\section{Kristina M.W. Mitchell}

Jonathan Martin

doi: https://doi.org/10.1017/S104909651800001X Published online by Cambridge University Press, 6 March 2018.

In our article "Gender Bias in Student Evaluations" (Mitchell and Martin 2018), we conducted statistical tests of the difference in proportions using as a sample size the total number of observations in our data. We also recognize that statistical tests using separate samples for the individual teaching evaluations submitted for the female and male instructors lead to different findings.

The results using this different sample size are reported in the revised tables 1 and 2 below.

While we recognize the results reported below do not meet conventional levels of statistical significance, the differences are in the hypothesized directions and in line with our conclusions. We strongly encourage scholars to continue this very important and timely line of research to provide additional analyses of potential gender biases in student evaluations. -

Table 1

(Revised): Content Analysis for Official University Course Evaluations

\begin{tabular}{|c|c|c|c|c|}
\hline Theme & $\begin{array}{l}\text { Professor } \\
\text { Martin } \\
\text { (Man) }\end{array}$ & $\begin{array}{l}\text { Professor } \\
\text { Mitchell } \\
\text { (Woman) }\end{array}$ & Difference & $\begin{array}{l}\text { Z-Score } \\
\text { (P- value) }\end{array}$ \\
\hline Personality & $4.3 \%$ & $15.6 \%$ & -11.2 & $1.4(.1714)$ \\
\hline Appearance & $0 \%$ & $0 \%$ & 0 & NA \\
\hline Entertainment & $15.2 \%$ & $32.2 \%$ & -17 & $1.5(.1327)$ \\
\hline $\begin{array}{l}\text { Intelligence/ } \\
\text { Competency }\end{array}$ & $13.0 \%$ & $11.0 \%$ & 2 & $0.2(.808)$ \\
\hline Incompetency & $0 \%$ & $0 \%$ & 0 & NA \\
\hline $\begin{array}{l}\text { Referred to as } \\
\text { "Professor" }\end{array}$ & $32.7 \%$ & $15.6 \%$ & 17.1 & $1.6(.1075)$ \\
\hline $\begin{array}{l}\text { Referred to as } \\
\text { "Teacher" }\end{array}$ & $15.2 \%$ & $24.4 \%$ & -9.2 & $0.9(.3808)$ \\
\hline $\mathbf{N}$ & 23 & 45 & & \\
\hline
\end{tabular}

Notes: $\mathrm{N}=68{ }^{*} \mathrm{p}<0.1 ;{ }^{* *} \mathrm{p}<0.05 ;{ }^{* * *} \mathrm{p}<0.001$
Table 2

(Revised): Content Analysis for Rate My Professors Comments

\begin{tabular}{|c|c|c|c|c|}
\hline Theme & $\begin{array}{l}\text { Professor } \\
\text { Martin } \\
\text { (Man) }\end{array}$ & $\begin{array}{l}\text { Professor } \\
\text { Mitchell } \\
\text { (Woman) }\end{array}$ & Difference & $\begin{array}{l}\text { Z-Score } \\
\text { (P- value) }\end{array}$ \\
\hline Personality & $11 \%$ & $20.9 \%$ & -9.9 & $0.7(.4917)$ \\
\hline Appearance & $0 \%$ & $10.6 \%$ & -10.6 & $1.0(.3063)$ \\
\hline Entertainment & $5.5 \%$ & $3.3 \%$ & 2.3 & $0.3(.7485)$ \\
\hline $\begin{array}{l}\text { Intelligence/ } \\
\text { Competency }\end{array}$ & $0 \%$ & $1.1 \%$ & -1.1 & $0.3(.7519)$ \\
\hline Incompetency & $0 \%$ & $6.6 \%$ & -6.6 & $0.8(.4279)$ \\
\hline $\begin{array}{l}\text { Referred to as } \\
\text { "Professor" }\end{array}$ & $22.2 \%$ & $22.0 \%$ & 0.3 & $0.0(.9895)$ \\
\hline $\begin{array}{l}\text { Referred to as } \\
\text { "Teacher" }\end{array}$ & $0 \%$ & $5.5 \%$ & -5.5 & $0.7(.4714)$ \\
\hline $\mathbf{N}$ & 9 & 45 & & \\
\hline
\end{tabular}

Notes: $N=54{ }^{*} p<0.1 ;{ }^{* *} p<0.05 ;{ }^{* *} p<0.001$

\section{REFERENCE}

Mitchell, Kristina M.W., and Jonathan Martin. 2018. "Gender Bias in Student Evaluations.” PS: Political Science E Politics 51 (3): 648-52. https://doi.org/10.1017/ S104909651800001X. 